ANNALES

POLONICI MATHEMATICI

LXVI (1997)

\title{
Analytic formulas for the hyperbolic distance between two contractions
}

\author{
by Ion Suciu (Bucharest) \\ Dedicated to the memory of Wtodzimierz Mlak
}

\begin{abstract}
In this paper we give some analytic formulas for the hyperbolic (Harnack) distance between two contractions which permit concrete computations in several situations, including the finite-dimensional case. The main consequence of these formulas is the proof of the Schwarz-Pick Lemma. It modifies those given in [13] by the avoidance of a general Schur type formula for contractive analytic functions, more exactly by reducing the case to the more manageable situation when the function takes as values strict contractions.
\end{abstract}

The Harnack equivalence was introduced in [11] and studied in many articles (cf. [1], [3], [5], [6], [16]).

The hyperbolic (Harnack) distance on the Harnack parts was introduced in [15] in a general context of completely positive maps from a subspace of a $C^{*}$-algebra into $B(H)$. The special case of the contractions was considered in [13], where the Schwarz-Pick Lemma for an operator-valued contractive analytic function defined on the open unit disc in the complex plane was proved.

In the present paper we give some analytic formulas for the hyperbolic (Harnack) distance between two contractions (Sections 2, 3) which permit concrete computations in several situations, including the finite-dimensional case. The main consequence of these formulas is the proof of the SchwarzPick Lemma given in Section 4. It modifies those given in [13] by the avoidance of a general Schur type formula for contractive analytic functions, more exactly by reducing the case to the more manageable situation when the function takes strict contractions as values.

1991 Mathematics Subject Classification: Primary 47A20, 47A45, 47A63; Secondary $30 \mathrm{C} 80$.

Key words and phrases: Harnack parts, hyperbolic distance, operator Schwarz-Pick Lemma. 
The paper uses as preliminaries elements of the Sz.-Nagy-Foias theory of contractions. Some of them are presented in Section 1, following mainly [4], [7] and [17].

1. Preliminaries. Let $H$ be a separable Hilbert space and $B(H)$ be the Banach space of all linear bounded operators on $H$. We shall denote by $B_{0}(H)$ the open unit ball in $B(H)$ and by $B_{1}(H)$ its closure. For a contraction $A \in B_{1}(H), D_{A}=\left[I-A^{*} A\right]^{1 / 2}$ is the defect operator of $A$ and $\mathcal{D}_{A}=\overline{D_{A}(H)}$ its defect space.

We shall denote by $\widetilde{U}_{A}$ acting on $\widetilde{K}_{A}$ the minimal unitary dilation of $A$ (cf. [17]). If $K_{A}=\bigvee_{n \geq 0} \widetilde{U}_{A}^{n} H, K_{* A}=\bigvee_{n \leq 0} \widetilde{U}_{A}^{n} H, U_{A}=\widetilde{U}_{A} \mid K_{A}$ then $U_{A}$ acting on $K_{A}$ is the minimal isometric dilation of $A$. We have

$$
\widetilde{K}_{A}=\left[K_{* A} \ominus H\right] \oplus H \oplus\left[K_{A} \ominus H\right] .
$$

For the minimal isometric dilation of $A$ we have the following matrix model (cf. [17]):

$$
K_{A}=H \oplus H^{2}\left(\mathcal{D}_{A}\right), \quad U_{A}=\left(\begin{array}{cc}
A & 0 \\
D_{A} & \mathcal{S}
\end{array}\right),
$$

where for a Hilbert space $\mathcal{E}, H^{2}(\mathcal{E})$ is the usual Hardy space and $\mathcal{S}$ is the shift operator on $H^{2}(\mathcal{E})$.

We shall adopt and use freely other notations and terminology from the Sz.-Nagy-Foiaş theory of contractions following the book [17]. Other terminology is from [4] and [7].

For example, if $\mathcal{E}$ and $\mathcal{F}$ are separable Hilbert spaces, we shall denote by $H^{\infty}(B(\mathcal{E}, \mathcal{F}))$ the Banach space of all bounded analytic functions defined on the unit disc $\mathbf{D}$ in the complex plane $\mathbb{C}$ with values bounded linear operators from $\mathcal{E}$ to $\mathcal{F}$. $H^{\infty}(B(\mathcal{E}))$ is $H^{\infty}(B(\mathcal{E}, \mathcal{E}))$ and $H_{1}^{\infty}(B(\mathcal{E}, \mathcal{F}))$ is the unit ball of $H^{\infty}(B(\mathcal{E}, \mathcal{F}))$. A function $\Theta \in H^{\infty}(B(\mathcal{E}, \mathcal{F}))$ has a.e. radial strong limit $\Theta\left(e^{i t}\right)$. Pointwise multiplication defines the bounded operators $\Theta$ from $H^{2}(\mathcal{E})$ into $H^{2}(\mathcal{F})$ and $\widetilde{\Theta}$ from $L^{2}(\mathcal{E})$ into $L^{2}(\mathcal{F}), \widetilde{\Theta}$ being the unique extention of $\Theta$ to an intertwining of the bilateral shifts on $L^{2}(\mathcal{E})$ and $L^{2}(\mathcal{F})$ respectively. We have

$$
\|\Theta\|=\|\widetilde{\Theta}\|=\|\Theta\|_{\infty}=\sup _{z \in \mathbf{D}}\|\Theta(z)\|=\underset{0 \leq t \leq 2 \pi}{\operatorname{ess} \sup }\left\|\Theta\left(e^{i t}\right)\right\| .
$$

An analytic function $\Omega$ from $\mathbf{D}$ into $B(\mathcal{E}, \mathcal{F})$ defined by

$$
\Omega(z)=\sum_{n=0}^{\infty} \Omega_{n} z^{n}
$$

will be called $L^{2}$-bounded if there exists a constant $M>0$ such that for any 
$a \in \mathcal{E}$ we have

$$
\sum_{n=0}^{\infty}\left\|\Omega_{n} a\right\|^{2} \leq M^{2}\|a\|^{2} .
$$

Clearly, (1.5) is equivalent to the fact that the formula

$$
(\Omega a)(z)=\Omega(z) a, \quad z \in \mathbf{D}, a \in \mathcal{E},
$$

defines a bounded operator $\Omega$ from $\mathcal{E}$ to $H^{2}(\mathcal{F})$, and $\|\Omega\| \leq M$.

2. Harnack parts and Harnack distance. We say that the contraction $A$ is Harnack dominated by the contraction $B$ (cf. [1]) if there exists a positive constant $c$ such that for any analytic polynomial $p$ satisfying $\operatorname{Re} p(z) \geq 0$ for $|z| \leq 1$ we have

$$
\operatorname{Re} p(A) \leq c^{2} \operatorname{Re} p(B) .
$$

Harnack domination is clearly a preorder relation on $B_{1}(H)$ and we shall call the equivalence relation induced by it Harnack equivalence (cf. [12]). The equivalence classes are called Harnack parts. For a contraction $A$ we shall denote by $\Delta(A)$ the Harnack part containing $A$. Clearly, $A$ and $B$ are Harnack equivalent if and only if there exists $a \geq 1$ such that

$$
\left(1 / a^{2}\right) \operatorname{Re} p(A) \leq \operatorname{Re} p(B) \leq a^{2} \operatorname{Re} p(A)
$$

for any analytic polynomial $p$ with positive real part on the unit disc $\mathbf{D}$.

Let us denote

$$
\omega_{H}(A, B)=\inf \{a \mid(2.2) \text { holds for } a\}
$$

and

$$
\delta_{H}(A, B)=\log \omega_{H}(A, B) .
$$

It is easy to see that $\omega_{H}(A, B)$ is symmetric in $A, B$ and $\delta_{H}(A, B)$ is a distance on each Harnack part $\Delta$ of $B_{1}(H)$. We shall call this distance the Harnack (hyperbolic) distance on $\Delta$ (cf. [15]).

In case $H=\mathbb{C}$ the only non-trivial (one-point) Harnack part of $B_{1}(H)$ is $\Delta(0)=\mathbf{D}$ and the Harnack distance on $\mathbf{D}$ coincides with the classical hyperbolic (Poincaré) distance $\delta$ on $\mathbf{D}$ defined by

$$
\delta(z, w)=\tanh ^{-1}\left(\frac{|z-w|}{|1-\bar{w} z|}\right), \quad z, w \in \mathbf{D} .
$$

It was shown in [12] that (2.1) is equivalent to the fact that there exists a bounded operator $S_{B, A}$ from $K_{B}$ to $K_{A}$ such that $\left\|S_{B, A}\right\| \leq c$ and for any $h_{0}, h_{1}, \ldots, h_{n} \in H$ we have

$$
S_{B, A} \sum_{j=0}^{n} U_{B}^{j} h_{j}=\sum_{j=0}^{n} U_{A}^{j} h_{j} .
$$


Clearly, in this case $S_{B, A}$ is the unique bounded operator from $K_{B}$ to $K_{A}$ which intertwines $U_{B}$ and $U_{A}$ and whose restriction to $H$ is the identity operator. $A$ is Harnack equivalent to $B$ if and only if $S_{B, A}$ is invertible. We have $S_{B, A}^{-1}=S_{A, B}$ and

$$
\omega_{H}(A, B)=\max \left\{\left\|S_{A, B}\right\|,\left\|S_{B, A}\right\|\right\} .
$$

It was shown in [3] that $A$ is Harnack equivalent to the null contraction on $H$ if and only if $A$ is a strict contraction, i.e. $\|A\|<1$. This means that $B_{0}$ is the Harnack part $\Delta\left(0_{H}\right)$ of the null contraction on $H$.

For $T \in B_{1}(H)$ and $0<r<1$ let us put $T_{r}=r T$.

TheOrem 1. For $A, B \in B_{1}(H)$ the following assertions are equivalent:

(i) $A$ is Harnack dominated by $B$.

(ii) $\sup _{0<r<1}\left\|S_{B_{r}, A_{r}}\right\|<\infty$.

If (i), and consequently (ii), holds then

$$
\left\|S_{B, A}\right\|=\sup _{0<r<1}\left\|S_{B_{r}, A_{r}}\right\| .
$$

Proof. For any $\lambda \in \mathbf{D}$, let $f(z, \lambda)=(1+\bar{\lambda} z) /(1-\bar{\lambda} z), z \in \mathbf{D}$. Since $\operatorname{Re} f(z, \lambda) \geq 0$ for $z \in \mathbf{D}$ and $f(z, \lambda)$ is in $\mathbf{D}$ a uniform limit of analytic polynomials with positive real part, it follows from (2.1) that

$$
\operatorname{Re} f(A, \lambda) \leq\left\|S_{B, A}\right\|^{2} \operatorname{Re} f(B, \lambda) .
$$

Since any analytic polynomial $p$ with positive real part can be written in the form

$$
p(z)=\int_{0}^{2 \pi} \frac{1+e^{-i t} z}{1-e^{-i t} z} d \mu(t)+i \alpha,
$$

where $\mu$ is a positive measure and $\alpha$ is a real constant, by (2.8) we have

$$
\begin{aligned}
\operatorname{Re} p\left(A_{r}\right) & =\int_{0}^{2 \pi} \operatorname{Re} f\left(A, r e^{i t}\right) d \mu(t) \\
& \leq\left\|S_{B, A}\right\|^{2} \int_{0}^{2 \pi} \operatorname{Re} f\left(B, r e^{i t}\right) d \mu(t) \leq\left\|S_{B, A}\right\|^{2} \operatorname{Re} p\left(B_{r}\right) .
\end{aligned}
$$

Hence for any $r, 0<r<1$, we have

$$
\left\|S_{B_{r}, A_{r}}\right\| \leq\left\|S_{B, A}\right\|
$$

and the implication (i) $\Rightarrow$ (ii) is proved.

Suppose now that

$$
\sup _{0<r<1}\left\|S_{B_{r}, A_{r}}\right\|=c<\infty
$$


For any $r, 0<r<1$, and any analytic polynomial $p$ with positive real part we have

$$
\operatorname{Re} p(r A)=\operatorname{Re} p\left(A_{r}\right) \leq\left\|S_{B_{r}, A_{r}}\right\|^{2} \operatorname{Re} p\left(B_{r}\right) \leq c^{2} \operatorname{Re} p\left(B_{r}\right)=c^{2} \operatorname{Re} p(r B) .
$$

Letting $r \rightarrow 1$ we obtain

$$
\operatorname{Re} p(A) \leq c^{2} \operatorname{Re} p(B) .
$$

Hence $A$ is Harnack dominated by $B$ and

$$
\left\|S_{B, A}\right\| \leq c=\sup _{0<r<1}\left\|S_{B_{r}, A_{r}}\right\| .
$$

From (2.11) and (2.12) we obtain (2.7) and the proof is complete.

Corollary 1. The contractions A, B are Harnack equivalent if and only if

$$
\sup _{0<r<1} \omega_{H}\left(A_{r}, B_{r}\right)<\infty
$$

If this is the case, we have

$$
\begin{aligned}
\omega_{H}(A, B) & =\sup _{0<r<1} \omega_{H}\left(A_{r}, B_{r}\right), \\
\delta_{H}(A, B) & =\sup _{0<r<1} \delta_{H}\left(A_{r}, B_{r}\right) .
\end{aligned}
$$

Corollary 2. For any $A, B \in B_{1}(H)$, both $\left\|S_{B_{r}, A_{r}}\right\|$ and $\omega_{H}\left(A_{r}, B_{r}\right)$ are increasing functions of $r$ on the interval $(0,1)$.

3. The analytic structure of the operator $S$. Having in mind the Sz.-Nagy-Foias structure (1.2) of the minimal isometric dilation, we look for a matrix form of the intertwining operator $S$. It will be given by a pair of analytic functions as in the following:

Theorem 2. The contraction A is Harnack dominated by the contraction $B$ if and only if there exist bounded operators $\Omega_{0}$ from $H$ to $\mathcal{D}_{B}$ and $\Theta_{0}$ from $\mathcal{D}_{A}$ to $\mathcal{D}_{B}$ such that:

(i) We have

$$
\begin{aligned}
& D_{B} \Omega_{0}=A^{*}-B^{*}, \\
& D_{B} \Theta_{0}=D_{A} .
\end{aligned}
$$

(ii) The formula

$$
\Omega(\lambda)=\Omega_{0}\left[I-\lambda A^{*}\right]^{-1}
$$

defines a $B\left(H, \mathcal{D}_{B}\right)$-valued $L^{2}$-bounded analytic function.

(iii) The formula

$$
\Theta(\lambda)=\Theta_{0}+\lambda \Omega_{0}\left[I-\lambda A^{*}\right]^{-1} D_{A}
$$

defines a $B\left(\mathcal{D}_{A}, \mathcal{D}_{B}\right)$-valued bounded analytic function. 
If this is the case then the bounded operator $S=S_{B, A}$ from $K_{B}=$ $H \oplus H^{2}\left(\mathcal{D}_{B}\right)$ into $K_{A}=H \oplus H^{2}\left(\mathcal{D}_{A}\right)$ satisfying $S \mid H=I_{H}$ and $S U_{B}=U_{A} S$ is given by

$$
S^{*}=\left(\begin{array}{cc}
I & 0 \\
\Omega & \Theta
\end{array}\right): \begin{gathered}
H \\
H^{2}\left(\mathcal{D}_{A}\right)
\end{gathered} \rightarrow \begin{gathered}
H \\
H^{2}\left(\mathcal{D}_{B}\right)
\end{gathered},
$$

where $\Omega$ and $\Theta$ are the operators from $H$ to $H^{2}\left(\mathcal{D}_{B}\right)$ and from $H^{2}\left(\mathcal{D}_{A}\right)$ to $H^{2}\left(\mathcal{D}_{B}\right)$ defined by

$$
\begin{array}{ll}
(\Omega h)(\lambda)=\Omega(\lambda) h, & h \in H, \lambda \in \mathbf{D}, \\
(\Theta f)(\lambda)=\Theta(\lambda) f(\lambda), \quad f \in H^{2}\left(\mathcal{D}_{B}\right), \lambda \in \mathbf{D} .
\end{array}
$$

Proof. Suppose $A$ is Harnack dominated by $B$ and let $S=S_{B, A}$ : $K_{B} \rightarrow K_{A}$ be the corresponding intertwining. Since $S \mid H=I_{H}$ we have

$$
S^{*}=\left(\begin{array}{cc}
I & 0 \\
\Omega & \Theta
\end{array}\right): \begin{gathered}
H \\
H^{2}\left(\mathcal{D}_{A}\right)
\end{gathered} \rightarrow \begin{gathered}
H \\
H^{2}\left(\mathcal{D}_{B}\right)
\end{gathered},
$$

where $\Omega$ is a bounded operator from $H$ to $H^{2}\left(\mathcal{D}_{B}\right)$ and $\Theta$ is a bounnded operator from $H^{2}\left(\mathcal{D}_{A}\right)$ to $H^{2}\left(\mathcal{D}_{B}\right)$.

From $S^{*} U_{A}^{*}=U_{B}^{*} S^{*}$ we obtain

$$
\left(\begin{array}{cc}
I & 0 \\
\Omega & \Theta
\end{array}\right)\left(\begin{array}{cc}
A^{*} & D_{A}^{0} \\
0 & \mathcal{S}^{*}
\end{array}\right)=\left(\begin{array}{cc}
B^{*} & D_{B}^{0} \\
0 & \mathcal{S}^{*}
\end{array}\right)\left(\begin{array}{cc}
I & 0 \\
\Omega & \Theta
\end{array}\right)
$$

where for a contraction $T$ on $H, D_{T}^{0}$ is the operator from $H^{2}\left(\mathcal{D}_{T}\right)$ into $H$ defined by

$$
D_{T}^{0} f=D_{T} f(0), \quad f \in H^{2}\left(\mathcal{D}_{T}\right) .
$$

We obtain

$$
\left(\begin{array}{cc}
A^{*} & D_{A}^{0} \\
\Omega A^{*} & \Omega D_{A}^{0}+\Theta \mathcal{S}^{*}
\end{array}\right)=\left(\begin{array}{cc}
B^{*}+D_{B}^{0} \Omega & D_{B}^{0} \Theta \\
\mathcal{S}^{*} \Omega & \mathcal{S}^{*} \Theta
\end{array}\right)
$$

Hence

$$
\begin{aligned}
A^{*} & =B^{*}+D_{B}^{0} \Omega, \\
\Omega A^{*} & =\mathcal{S}^{*} \Omega, \\
D_{A}^{0} & =D_{B}^{0} \Theta, \\
\Omega D_{A}^{0} & =\mathcal{S}^{*} \Theta-\Theta \mathcal{S}^{*} .
\end{aligned}
$$

Let $\Omega_{0}$ be the operator from $H$ to $\mathcal{D}_{B}$ defined by

$$
\Omega_{0} h=(\Omega h)(0) \text {. }
$$

Since

$$
\left\|\Omega_{0} h\right\|_{\mathcal{D}_{B}}^{2}=\|(\Omega h)(0)\|_{\mathcal{D}_{B}}^{2} \leq\|\Omega h\|_{H^{2}\left(\mathcal{D}_{B}\right)}^{2} \leq\|\Omega\|^{2}\|h\|^{2},
$$


it results that $\Omega_{0}$ is bounded. From (3.6) we obtain

$$
D_{B} \Omega_{0} h=D_{B}(\Omega h)(0)=D_{B}^{0} \Omega h=\left(A^{*}-B^{*}\right) h,
$$

for any $h \in H$. Hence

$$
D_{B} \Omega_{0}=A^{*}-B^{*}
$$

Using (3.7) we obtain

$$
(1 / \lambda)[(\Omega h)(\lambda)-(\Omega h)(0)]=\left(\mathcal{S}^{*} \Omega h\right)(\lambda)=\left(\Omega A^{*} h\right)(\lambda)
$$

for any $h \in H$ and $\lambda \in \mathbf{D}, \lambda \neq 0$. Thus

$$
(\Omega h)(\lambda)=\Omega_{0} h+\lambda\left(\Omega A^{*} h\right)(\lambda),
$$

which implies

$$
(\Omega h)(\lambda)=\Omega_{0}\left[I-\lambda A^{*}\right]^{-1} h .
$$

It results that $\Omega$ is given by the multiplication on $H$ with the $L^{2}$-bounded analytic function defined on $\mathbf{D}$ by

$$
\Omega(\lambda)=\Omega_{0}\left[I-\lambda A^{*}\right]^{-1} .
$$

Setting

$$
\Theta_{0} g=(\Theta g)(0), \quad g \in \mathcal{D}_{A},
$$

we obtain a bounded operator $\Theta_{0}$ from $\mathcal{D}_{A}$ to $\mathcal{D}_{B}$. From (3.8) it results that

$$
D_{B} \Theta_{0}=D_{A},
$$

and from (3.9) we have

$$
\begin{aligned}
(1 / \lambda)\left[(\Theta g)(\lambda)-\Theta_{0} g\right] & =(1 / \lambda)[(\Theta g)(\lambda)-(\Theta g)(0)]=\left(\mathcal{S}^{*} \Theta g\right)(\lambda) \\
& =\left(\left[\mathcal{S}^{*} \Theta-\Theta \mathcal{S}^{*}\right] g\right)(\lambda)=\left(\Omega D_{A} g\right)(\lambda)=\Omega(\lambda) D_{A} g
\end{aligned}
$$

for any $g \in D_{B}$ and $\lambda \in \mathbf{D}, \lambda \neq 0$. Hence

$$
(\Theta g)(\lambda)=\Theta_{0} g+\lambda \Omega(\lambda) D_{A} g, \quad g \in D_{B}, \lambda \in \mathbf{D} .
$$

It results that $\Theta$ is the operator from $H^{2}\left(\mathcal{D}_{A}\right)$ into $H^{2}\left(\mathcal{D}_{B}\right)$ given by the pointwise multiplication with the bounded analytic function

$$
\Theta(\lambda)=\Theta_{0}+\lambda \Omega_{0}\left[I-\lambda A^{*}\right]^{-1} D_{A} .
$$

In this way we produced $\Omega_{0}, \Theta_{0}$ such that (i)-(iii) are satisfied and $S$ has the form (3.5).

Suppose now there exist $\Omega_{0}, \Theta_{0}$ such that (i)-(iii) hold and let $S$ be defined by (3.5). Clearly we have $A^{*}=B^{*}+D_{B}^{0} \Omega$ and $D_{A}^{0}=D_{B}^{0} \Theta$. Since for any $h \in H$ we have

$$
\begin{aligned}
\left(\mathcal{S}^{*} \Omega h\right)(\lambda) & =(1 / \lambda)[(\Omega h)(\lambda)-(\Omega h)(0)]=(1 / \lambda)\left(\Omega_{0}\left[I-\lambda A^{*}\right]^{-1} h-\Omega_{0} h\right) \\
& =(1 / \lambda)\left(\Omega_{0}-\Omega_{0}\left[I-\lambda A^{*}\right]\right)\left[I-\lambda A^{*}\right]^{-1} h=\Omega_{0} A^{*}\left[I-\lambda A^{*}\right]^{-1} h \\
& =\Omega_{0}\left[I-\lambda A^{*}\right]^{-1} A^{*} h=\left(\Omega A^{*} h\right)(\lambda)
\end{aligned}
$$


and for any $f \in H^{2}\left(\mathcal{D}_{B}\right)$ we have

$$
\begin{aligned}
\left(\mathcal{S}^{*} \Theta f-\Theta \mathcal{S}^{*} f\right)(\lambda)= & (1 / \lambda)\left[\Theta(\lambda) f(\lambda)-\Theta_{0} f(0)\right]-\Theta(\lambda)(1 / \lambda)[f(\lambda)-f(0)] \\
= & (1 / \lambda)\left[\Theta_{0} f(\lambda)+\lambda \Omega(\lambda) D_{A} f(\lambda)-\Theta_{0} f(0)\right] \\
& -(1 / \lambda) \Theta_{0}[f(\lambda)-f(0)]-\Omega(\lambda) D_{A}[f(\lambda)-f(0)] \\
= & (1 / \lambda) \Theta_{0}[f(\lambda)-f(0)]+\Omega(\lambda) f(\lambda) \\
& -(1 / \lambda) \Theta_{0}[f(\lambda)-f(0)]-\Omega(\lambda) D_{A}[f(\lambda)-f(0)] \\
= & \Omega(\lambda) D_{A} f(0)=\left(\Omega D_{A}^{0} f\right)(\lambda),
\end{aligned}
$$

it results that the relations (3.6)-(3.9) are fulfilled. These clearly imply $S^{*} U_{A}^{*}=U_{B}^{*} S^{*}$. Since $S \mid H=I_{H}$ we proved that $A$ is Harnack dominated by $B$.

The proof of Theorem 2 is complete.

Let $\Omega_{B, A}=\Omega, \Theta_{B, A}=\Theta$ if $\Omega, \Theta$ appear in the form (3.5) of $S=S_{B, A}$.

Corollary 3. If $A$ is Harnack dominated by $C$ and $C$ is Harnack dominated by $B$, then $A$ is Harnack dominated by $B$ and

$$
\Theta_{B, A}=\Theta_{B, C} \Theta_{C, A} .
$$

When $A$ is Harnack dominated by $B$, they are Harnack equivalent if and only if the corresponding $\Theta_{B, A}$ is invertible. In this case we have

$$
\Theta_{A, B}=\Theta_{B, A}^{-1} .
$$

Corollary 4. The contraction $A$ is Harnack dominated by the null contraction if and only if the spectrum $\sigma(A)$ of $A$ is contained in $\mathbf{D}$. Furthermore, $A$ is Harnack equivalent to the null contraction if and only if $\|A\|<1$.

Proof. If $A$ is Harnack dominated by $B=0$ then $\Omega_{0}=A^{*}, \Theta_{0}=D_{A}$ and

$$
\Omega_{0, A}=A^{*}\left[I-\lambda A^{*}\right]^{-1}, \quad \Theta_{0, A}=\left[I-\lambda A^{*}\right]^{-1} D_{A} .
$$

Hence $\Theta_{B, A}$ is bounded if and only if $\sigma(A) \subset \mathbf{D}$. For the second assertion, $A$ is Harnack equivalent to 0 if and only if $\Theta_{0}=D_{A}$ is invertible, i.e. if and only if $\|A\|<1$.

Corollary 5. Suppose $A, B$ are strict contractions. Then $A$ and $B$ are Harnack equivalent and

$$
\Theta_{B, A}(\lambda)=D_{B}^{-1}\left[I-\lambda B^{*}\right]\left[I-\lambda A^{*}\right]^{-1} D_{A} .
$$

In computing the Harnack distance beetwen two Harnack equivalent contractions $A$ and $B$ we are interested in $\left\|S_{A, B}\right\|$ and $\left\|S_{B, A}\right\|$. We have the following: 
Theorem 3. Let $A$ and $B$ be strict contractions on $H$. Then

$$
\left\|S_{B, A}\right\|=\left\|\Theta_{B, A}\right\|_{\infty}=\sup _{0 \leq t \leq 2 \pi}\left\|D_{B}^{-1}\left[I-e^{i t} B^{*}\right]\left[I-e^{i t} A^{*}\right]^{-1} D_{A}\right\|,
$$

and

$$
\omega_{H}(A, B)=\max \left(\left\|\Theta_{A, B}\right\|_{\infty},\left\|\Theta_{B, A}\right\|_{\infty}\right) .
$$

Proof. Let $S=S_{B, A}, \Theta=\Theta_{B, A}$ be as in Theorem 2. Extend $S$ to an intertwining $\widetilde{S}$ of $\widetilde{U}_{B}$ with $\widetilde{U}_{A}$. This means $\widetilde{S}: \widetilde{K}_{A} \rightarrow \widetilde{K}_{B}, \widetilde{U}_{A} \widetilde{S}=\widetilde{S}_{B}$ and $\widetilde{S} \mid K_{B}=S$. Clearly, $\widetilde{S} K_{B} \subset K_{A}, \widetilde{S} K_{* B} \subset K_{* A}$. From (1.1) it results that $\widetilde{S}^{*}\left[K_{A} \ominus H\right] \subset K_{B} \ominus H$. Hence $\widetilde{S}^{*}\left|K_{B} \ominus H=S^{*}\right| K_{B} \ominus H$.

Since $A$ and $B$ are strict contractions we can identify their unitary dilations with the bilateral shift on $L^{2}(H)$. In this identification $K_{A}$ and $K_{B}$ become subspaces in $L^{2}(H)$ such that $K_{A} \ominus H$ and $K_{B} \ominus H$ become $H^{2}(H)$. From (3.5) we obtain

$$
\widetilde{S}^{*} \mid H^{2}(H)=\Theta .
$$

It results that in this model $\widetilde{S}^{*}$ is given by the pointwise multiplication by $\Theta$ on $L^{2}(H)$. Consequently,

$$
\left\|\widetilde{S}^{*}\right\|=\|\Theta\|_{\infty}
$$

Since $\|S\|=\|\widetilde{S}\|=\left\|\widetilde{S}^{*}\right\|$, using (3.16) and (3.19) we obtain

$$
\begin{aligned}
\left\|S_{B, A}\right\| & =\left\|\Theta_{B, A}\right\|_{\infty}=\sup _{\lambda \in \mathbf{D}}\left\|D_{B}^{-1}\left[I-\lambda B^{*}\right]\left[I-\lambda A^{*}\right]^{-1} D_{A}\right\| \\
& =\sup _{0 \leq t \leq 2 \pi}\left\|D_{B}^{-1}\left[I-e^{i t} B^{*}\right]\left[I-e^{i t} A^{*}\right]^{-1} D_{A}\right\| .
\end{aligned}
$$

We also have

$$
\begin{aligned}
\omega_{H}(A, B)= & \max \left\{\left\|\Theta_{A, B}\right\|_{\infty},\left\|\Theta_{B, A}\right\|_{\infty}\right\} \\
= & \max \left\{\sup _{0 \leq t \leq<2 \pi}\left\|D_{B}^{-1}\left[I-e^{i t} B^{*}\right]\left[I-e^{i t} A^{*}\right]^{-1} D_{A}\right\|,\right. \\
& \left.\sup _{0 \leq t<2 \pi}\left\|D_{A}^{-1}\left[I-e^{i t} A^{*}\right]\left[I-e^{i t} B^{*}\right]^{-1} D_{B}\right\|\right\}
\end{aligned}
$$

and the theorem is proved.

Corollary 6. For any strict contraction $A$ we have

$$
\frac{1}{2} \log \frac{1}{1-\|A\|} \leq \delta_{H}(0, A) \leq \frac{1}{2} \log \frac{1+\|A\|}{1-\|A\|} .
$$

If $A$ is a normal strict contraction then

$$
\delta_{H}(0, A)=\frac{1}{2} \log \frac{1+\|A\|}{1-\|A\|} .
$$


Pr o of. From (3.16) we have

$$
\Theta_{0, A}(\lambda)=\left[I-\lambda A^{*}\right]^{-1} D_{A}, \quad \Theta_{A, 0}(\lambda)=D_{A}^{-1}\left[I-\lambda A^{*}\right] .
$$

Hence

$$
\frac{1}{[1-\|A\|]^{1 / 2}} \leq\left\|\Theta_{A, 0}\right\|_{\infty}, \quad\left\|\Theta_{0, A}\right\|_{\infty} \leq \frac{[1+\|A\|]^{1 / 2}}{[1-\|A\|]^{1 / 2}} .
$$

If $A$ is a normal strict contraction then

$$
\left\|\Theta_{A, 0}\right\|_{\infty}=\sup _{0 \leq t \leq 2 \pi} \sup _{|z| \leq\|A\|} \frac{\left|1-e^{i t} z\right|}{\left[1-|z|^{2}\right]^{1 / 2}}=\frac{[1+\|A\|]^{1 / 2}}{[1-\|A\|]^{1 / 2}} .
$$

Corollary 7. For any two contractions $A, B$ which are Harnack equivalent we have

$$
\begin{gathered}
\omega_{H}(A, B)=\max \left\{\sup _{r} \sup _{t} \|\left[I-r^{2} A^{*} A\right]^{-1 / 2}\left[I-e^{i t} r A^{*}\right]\right. \\
\cdot\left[I-e^{i t} r B^{*}\right]^{-1}\left[I-r^{2} B^{*} B\right]^{1 / 2} \|, \\
\left.\sup _{r} \sup _{t}\left\|\left[I-r^{2} B^{*} B\right]^{-1 / 2}\left[I-e^{i t} r B^{*}\right]\left[I-e^{i t} r A^{*}\right]^{-1}\left[I-r^{2} A^{*} A\right]^{1 / 2}\right\|\right\} .
\end{gathered}
$$

4. Schwarz-Pick Lemma with respect to the Harnack distance. Using the formulas for the Harnack distance obtained in the previous sections, we shall prove the following Schwarz-Pick Lemma for operator-valued contractive analytic functions defined in the unit disc of complex plane.

Theorem 4. Let $F: \mathbf{D} \rightarrow B(H)$ be a contractive analytic function. For any $z_{1}, z_{2} \in \mathbf{D}$ the contractions $F\left(z_{1}\right), F\left(z_{2}\right)$ are Harnack equivalent and

$$
\delta_{H}\left(F\left(z_{1}\right), F\left(z_{2}\right)\right) \leq \delta\left(z_{1}, z_{2}\right)
$$

Proof. We can suppose $z_{1}=0$. Indeed, if for any function $G$ in $H_{1}^{\infty}(B(H))$ and $z_{0} \in \mathbf{D}$ we know that $G(0)$ and $G\left(z_{0}\right)$ are Harnack equivalent and

$$
\delta_{H}\left(G(0), G\left(z_{0}\right)\right) \leq \delta\left(0, z_{0}\right)
$$

then taking $\omega$ to be the Möbius transform

$$
\omega(z)=\frac{z+z_{1}}{1+\bar{z}_{1} z}
$$

and $G(z)=F(\omega(z)), z_{0}=\omega^{-1}\left(z_{2}\right)$ we have $G(0)=F(\omega(0))=F\left(z_{1}\right)$ and $G\left(z_{0}\right)=F\left(\omega\left(z_{0}\right)\right)=F\left(z_{2}\right)$. Hence

$$
\delta_{H}\left(F\left(z_{1}\right), F\left(z_{2}\right)\right)=\delta_{H}\left(G(0), G\left(z_{0}\right)\right) \leq \delta\left(0, z_{0}\right)=\delta\left(z_{1}, z_{2}\right)
$$

because the Poincaré distance is invariant under Möbius transformations.

We can also suppose that $\|F(z)\|<1$ for any $z \in \mathbf{D}$. Indeed, suppose that (4.1) holds for such a function. Setting $F(0)=A, F\left(z_{0}\right)=B$ for arbitrary 
$F$ in $H_{1}^{\infty}$, and $F_{r}=r F$ for any $r, 0<r<1$, we obtain $\left\|F_{r}(z)\right\| \leq r<1$, $F_{r}(0)=r A=A_{r}, F_{r}\left(z_{0}\right)=r B=B_{r}$ and consequently

$$
\delta_{H}\left(A_{r}, B_{r}\right)=\delta_{H}\left(F_{r}(0), F_{r}\left(z_{0}\right)\right) \leq \delta\left(0, z_{0}\right) .
$$

Hence

$$
\sup _{0<r<1} \delta_{H}\left(A_{r}, B_{r}\right) \leq \delta\left(0, z_{0}\right)<\infty .
$$

From Corollary 2 it results that $A$ and $B$ are Harnack equivalent and

$$
\delta_{H}(A, B)=\sup _{0<r<1} \delta_{H}\left(A_{r}, B_{r}\right) \leq \delta\left(0, z_{0}\right) .
$$

Suppose now that $F \in H_{1}^{\infty}(B(H))$ is such that $\|F(z)\|<1$ for any $z \in \mathbf{D}$. Let $z_{0} \in \mathbf{D}$ and $A=F(0), B=F\left(z_{0}\right) . \quad A$ and $B$ being strict contractions, they are Harnack equivalent. Let $G$ be the function defined on $\mathbf{D}$ by

$$
G(z)=-A+D_{A^{*}} F(z)\left[I-A^{*} F(z)\right]^{-1} D_{A}, \quad z \in \mathbf{D} .
$$

We have $G(0)=0$. Using the fact that the operator matrix

$$
\left(\begin{array}{cc}
-A & D_{A^{*}} \\
D_{A} & A^{*}
\end{array}\right)
$$

is unitary and remarking that

$$
\begin{aligned}
& \left(\begin{array}{cc}
-A & D_{A^{*}} \\
D_{A} & A^{*}
\end{array}\right)\left(\begin{array}{c}
h \\
F(z)\left[I-A^{*} F(z)\right]^{-1} D_{A} h
\end{array}\right) \\
& =\left(\begin{array}{c}
G(z) h \\
D_{A} h+A^{*} F(z)\left[I-A^{*} F(z)\right]^{-1} D_{A} h
\end{array}\right),
\end{aligned}
$$

we obtain

$$
\begin{aligned}
& \|G(z) h\|^{2} \\
& =\|h\|^{2}+\left\|F(z)\left[I-A^{*} F(z)\right]^{-1} D_{A} h\right\|^{2} \\
& \quad-\left\|D_{A} h+A^{*} F(z)\left[I-A^{*} F(z)\right]^{-1} D_{A} h\right\|^{2} \\
& =\|h\|^{2}-\left(\left\|\left[I-A^{*} F(z)\right]^{-1} D_{A} h\right\|^{2}-\left\|F(z)\left[I-A^{*} F(z)\right]^{-1} D_{A} h\right\|^{2}\right) \leq\|h\|^{2},
\end{aligned}
$$

for any $z \in \mathbf{D}$ and $h \in H$.

Hence $G(z)$ is contractive and $G(0)=0$. Then $G(z)=z F_{1}(z)$ with $F_{1}$ analytic in D. Since for any $r, 0<r<1$, and any $z, 0<|z| \leq r<1$, we have

$$
\left\|F_{1}(z)\right\|=\|G(z) / z\| \leq \sup _{|z|=r}\|G(z)\| / r \leq 1 / r
$$

it results that $\left\|F_{1}(z)\right\| \leq 1$ for any $z \in \mathbf{D}$, i.e. $F_{1} \in H_{1}^{\infty}(B(H))$. Also,

$$
\begin{aligned}
G(z) & =D_{A^{*}}^{-1}\left\{-A\left[I-A^{*} F(z)\right]+\left[I-A A^{*}\right] F(z)\right\}\left[I-A^{*} F(z)\right]^{-1} D_{A} \\
& =D_{A^{*}}^{-1}[F(z)-A]\left[I-A^{*} F(z)\right]^{-1} D_{A} .
\end{aligned}
$$


Hence

$$
F(z)=A+D_{A^{*}} G(z)\left[I+A^{*} G(z)\right]^{-1} D_{A}
$$

and from $G(z)=z F_{1}(z)$ we obtain

$$
F(z)=A+z D_{A^{*}} F_{1}(z)\left[I+z A^{*} F_{1}(z)\right]^{-1} D_{A}, \quad z \in \mathbf{D},
$$

with $F_{1} \in H_{1}^{\infty}(B(H))$.

Let us define

$$
C=F_{1}\left(z_{0}\right)
$$

we have $\|C\| \leq 1$ and from $F\left(z_{0}\right)=B$ we obtain

$$
B=A+z_{0} D_{A^{*}} C\left[I+z_{0} A^{*} C\right]^{-1} D_{A} .
$$

Using again the fact that the operator matrix

$$
\left(\begin{array}{cc}
A & D_{A^{*}} \\
A & -A^{*}
\end{array}\right)
$$

is unitary and (4.5) we obtain

$$
\begin{aligned}
\|B h\|^{2}= & \|h\|^{2}+\left\|z_{0} C\left[I+z_{0} A^{*} C\right]^{-1} D_{A} h\right\|^{2} \\
& -\left\|D_{A} h-z_{0} A^{*} C\left[I+z_{0} C\right]^{-1} D_{A} h\right\|^{2} \\
= & \|h\|^{2}+\left|z_{0}\right|^{2}\left\|C\left[I+z_{0} A^{*} C\right]^{-1} D_{A} h\right\|^{2}-\left\|\left[I+z_{0} A^{*} C\right]^{-1} D_{A} h\right\|^{*} .
\end{aligned}
$$

Hence

$$
\left\|D_{B} h\right\|^{2}=\left\|\left[I+z_{0} A^{*} C\right]^{-1} D_{A} h\right\|^{2}-\left|z_{0}\right|^{2}\left\|C\left[I+z_{0} A^{*} C\right]^{-1} D_{A} h\right\|^{2} .
$$

Writing $\varrho=\|C\|$, we have

$$
\left\|\left[I+z_{0} A^{*} C\right]^{-1} D_{A} h\right\|^{2} \leq \frac{1}{1-\left|z_{0}\right|^{2} \varrho^{2}}\left\|D_{B} h\right\|^{2} .
$$

Let now $\Theta=\Theta_{B, A}$ correspond as in Theorem 2 to the Harnack equivalent contractions $A$ and $B$. Since $A, B$ are strict contractions, according to (3.16) we have

$$
\Theta(\lambda)=\Theta_{B, A}(\lambda)=D_{B}^{-1}\left[I-\lambda B^{*}\right]\left[I-\lambda A^{*}\right]^{-1} D_{A}, \quad \lambda \in \mathbf{D} .
$$

Using (4.5) we obtain

$$
\begin{aligned}
\Theta(\lambda) & =D_{B}^{-1}\left\{I-\lambda A^{*}-\lambda \bar{z}_{0} D_{A}\left[I+\bar{z}_{0} C^{*} A\right]^{-1} C^{*} D_{A^{*}}\right\}\left[I-\lambda A^{*}\right]^{-1} D_{A} \\
& =D_{B}^{-1} D_{A}-\lambda \bar{z}_{0} D_{B}^{-1} D_{A}\left[I+\bar{z}_{0} C^{*} A\right]^{-1} C^{*} D_{A^{*}}\left[I-\lambda A^{*}\right]^{-1} D_{A} \\
& =D_{B}^{-1} D_{A}\left[I+\bar{z}_{0} C^{*} A\right]^{-1}\left\{I+\bar{z}_{0} C^{*} A-\lambda \bar{z}_{0} C^{*} D_{A^{*}}\left[I-\lambda A^{*}\right]^{-1} D_{A}\right\} \\
& =D_{B}^{-1} D_{A}\left[I+\bar{z}_{0} C^{*} A\right]^{-1}\left\{I+\bar{z}_{0} C^{*}\left(A-\lambda D_{A^{*}}\left[I-\lambda A^{*}\right]^{-1} D_{A}\right)\right\} .
\end{aligned}
$$

Hence

$$
\Theta(\lambda)=D_{B}^{-1} D_{A}\left[I+\bar{z}_{0} C^{*} A\right]^{-1}\left[I-\bar{z}_{0} C^{*} \Theta_{A}(\lambda)\right], \quad \lambda \in \mathbf{D},
$$


where

$$
\Theta_{A}(\lambda)=-A+\lambda D_{A^{*}}\left[I-\lambda A^{*}\right]^{-1} D_{A}
$$

is the characteristic function of $A$.

Using (4.6) and (4.7) and the fact that the characteristic function is contractive we obtain

$$
\begin{aligned}
\left\|\Theta(\lambda)^{*} h\right\|^{2} & =\left\|\left[I-z_{0} \Theta_{A}(\lambda)^{*} C\right]\left[I+z_{0} A^{*} C\right]^{-1} D_{A} D_{B}^{-1} h\right\|^{2} \\
& \leq \frac{\left[1+\left|z_{0}\right| \varrho\right]^{2}}{1-\left|z_{0}\right|^{2} \varrho^{2}}\|h\|^{2}=\frac{1+\left|z_{0}\right| \varrho}{1-\left|z_{0}\right| \varrho}\|h\|^{2} \leq \frac{1+\left|z_{0}\right|}{1-\left|z_{0}\right|}\|h\|^{2},
\end{aligned}
$$

for any $h \in H$ and $\lambda \in \mathbf{D}$.

From Theorem 3 it results that

$$
\left\|S_{B, A}\right\|=\sup _{\lambda \in \mathbf{D}}\left\|\Theta_{B, A}(\lambda)\right\|=\sup _{\lambda \in \mathbf{D}}\left\|\Theta(\lambda)^{*}\right\| \leq \frac{\left[1+\left|z_{0}\right|\right]^{1 / 2}}{\left[1-\left|z_{0}\right|\right]^{1 / 2}} .
$$

By symmetry we obtain

$$
\left\|S_{A, B}\right\| \leq \frac{\left[1+\left|z_{0}\right|\right]^{1 / 2}}{\left[1-\left|z_{0}\right|\right]^{1 / 2}}
$$

Thus

Hence

$$
\omega_{H}(A, B)=\max \left\{\left\|S_{A, B}\right\|,\left\|S_{B, A}\right\|\right\} \leq \frac{\left[1+\left|z_{0}\right|\right]^{1 / 2}}{\left[1-\left|z_{0}\right|\right]^{1 / 2}}
$$

$$
\delta_{H}(A, B) \leq \frac{1}{2} \log \frac{1+\left|z_{0}\right|}{1-\left|z_{0}\right|}=\delta\left(0, z_{0}\right) .
$$

Theorem 4 is completely proved.

\section{References}

[1] T. Ando, I. Suciu and D. Timotin, Characterization of some Harnack parts of contractions, J. Operator Theory 2 (1979), 233-245.

[2] S. Dineen, The Schwarz Lemma, Clarendon Press, Oxford, 1989.

[3] C. Foiaş, On Harnack parts of contractions, Rev. Roumaine Math. Pures Appl. 19 (1974), 314-318.

[4] C. Foiaş and A. E. Frazho, The Commutant Lifting Aproach to Interpolation Problems, Oper. Theory Adv. Appl. 44, Birkhäuser, Basel, 1990.

[5] V. A. Khatskevich, Yu. L. Shmul'yan and V. S. Shul'man, Equivalent contractions, Dokl. Akad. Nauk SSSR 278 (1) (1984), 47-49 (in Russian); English transl.: Soviet Math. Dokl. 30 (2) (1984), 338-340.

[6] -, -, -, Pre-orders and equivalences in the operator ball, Sibirsk. Mat. Zh. 32 (3) (1991) (in Russian); English transl.: Siberian Math. J. 32 (3) (1991), 496-506.

[7] W. Mlak, Hilbert Spaces and Operator Theory, PWN-Kluwer, Warszawa-Dordrecht, 1991.

[8] G. Pick, Über die Beschränkungen analytischer Funktionen, welche durch vorgegebene Funktionswerte bewirkt werden, Math. Ann. 77 (1916), 7-23. 
[9] H. A. Schwarz, Zur Theorie der Abbildung, in: Gesammelte Mathematische Abhandlungen, Band II, Springer, Berlin, 1890, 108-132.

[10] S. Stoilow, Theory of Functions of a Complex Variable, I, II, Editura Academiei, Bucureşti, 1954, 1958 (in Romanian).

[11] I. Suciu, Harnack inequalities for a functional calculus, in: Hilbert Space Operators and Operator Algebras (Proc. Internat. Conf., Tihany, 1970), Colloq. Math. Soc. János Bolyai 5, North-Holland, Amsterdam, 1972, 449-511.

[12] - Analytic relations between functional models for contractions, Acta Sci. Math. (Szeged) 33 (1973), 359-365.

[13] -, Schwarz Lemma for operator contractive analytic functions, preprint IMAR No. 5, 1992.

[14] - The Kobayashi distance between two contractions, in: Oper. Theory Adv. Appl. 61, Birkhäuser, Basel, 1993, 189-200.

[15] I. Suciu and I. Valuşescu, On the hyperbolic metric on Harnack parts, Studia Math. 55 (1976), 97-109.

[16] N. Suciu, On Harnack ordering of contractions, Bull. Acad. Polon. Sci. Sér. Sci. Math. 27 (1979), 467-471.

[17] B. Sz.-Nagy and C. Foias, Harmonic Analysis of Operators on Hilbert Space, North-Holland-American Elsevier-Akademiai Kiadó, Amsterdam-New York-Budapest, 1970.

Institute of Mathematics

Romanian Academy

P.O. Box 1-764

RO-70700, Bucharest, Romania

E-mail: isuciu@roimar.imar.ro 